stammer is of the blocking, explosive type he should see a speech therapist, and should do so in any case if the stammer does not clear up within the next few months.

\section{Arterial Disease}

Q. -What drugs have any effect on endarteritis or atheroma in a mnn of 62 with normal blood pressure and electrocardiogram, and with a negative Wassermann reaction? He has pain over the carotid and bronchial arteries but negligible angina.

A.-Points which need to be considered are: the aetiology, position, and extent of the endarteritis, the location of the atheroma, and whether the pain is spontaneous or occurs on pressure or with exercise. Unless endarteritis is due to syphilis or chronic suppurative lesions, we know of no drug that will affect it. Similarly, there is no known therapeutic agent for atheroma. The circulation can be improved by coronary vasodilators and undue cardiac strain avoided by limiting exercise to the point of anginal pain or breathlessness.

\section{Delousing Heads by "T.I.F.A."}

Q.-I recently read an American article describing the Todd insecticide fog-applicator ("T.I.F.A."). Could you tell me if anything comparable to this machine exists in this country, and, if so, its approximate cost? I contend that children with pediculosis capitis might be passed through a room containing a suitable proportion of D.D.T. fog applied with the T.I.F.A., or the like, at regular intervals. Could I have your advice?

A.- The T.I.F.A. fog insecticide disperser has been tested in this country but, so far as we are aware, is not manufactured here. It is a rather wasteful method of dispersing very large quantities of insecticide rapidly and over large areas. It might have advantages in large-scale field treatments, where, for example, it is desired to eradicate tsetse flies from large areas of African bush or to destroy rapidly all the mosquitoes in a small town or a large camp site. It is difficult to see why one should employ such a large, elaborate machine for the exceedingly simple procedure of applying insecticide to verminous heads. Several excellent preparations are available, such as "lethane special " (British Medical Journal, 1942, 1, 464), D.D.T. emulsion (ibid., 1945, 1, 409), or gamma-B.H.C. (Med. Officer, March 20, 1948). All of these are better applied by hand than by a fog in the air.

\section{Care of the Hair and Scalp}

Q.-(a) What happens to the hair and scalp if left unwashed for a long time, say two years? (b) Is water or washing inimical to the health of the hair? (c) What are the most healthy materials for washing hair? (d) What is the best treatment for dandruff?

A.- (a) Provided brushes and combs are washed frequently, and the hair is well brushed daily, a healthy scalp and hair need not be washed for years.

(b) Hard water and alkaline soaps are not good for the hair ; with proper care there is no objection to frequent washing. This is not advisable except where the hair is very oily or the scalp has much dandruff.

(c) Rain water, soft water, and any good soap are best for the purpose. For the many methods which are harmless consult a book on dermatology.

(d) Dandruff (pityriasis capitis) is too big a problem to answer without examining the patient. If left alone, the next stagespityriasis steatoides and circinata, with redness, exudation, and crusting-may develop. For simple dandruff use salicylic acid, gr. $8\left(0.52 \mathrm{~g}\right.$.) and/or liquor carbonis detergens, $\frac{1}{2}$ to $1 \mathrm{drachm}$ $(1.8$ to $3.5 \mathrm{ml}$.) to the ounce $(28.4 \mathrm{ml}$.) of industrial spirit, and water, equal parts. Some respond better to mercurial preparations. For severe cases the same drugs may be used in ointment form.

\section{Penicillin and Tuberculosis}

Q.-Tuberculous abscesses after penicillin injections have been described. Is the incidence of tuberculous infection following penicillin injection higher than after other types of injection? Does penicillin favour the growth of Mycobacterium tuberculosis?

A.- When tuberculous abscesses have resulted from therapeutic injections they have usually been due to the use of a contaminated syringe. The kind of material injected is likely to be of little consequence if it contains living tubercle bacilli, and presumably this occurrence is not so common that the incidence of tuberculous infection after penicillin and other $\bar{z}$ injections can be compared statistically. According to Ungar $\mathbb{Q}$ and Muggleton (J. Path. Bact., 1946, 58, 501) penicillin stimu- $C$ lates the growth of Myco. tuberculosis in culture. Rivière, Thély, and Gautron (C. R. Acad. Sci., Paris, 1947, 224, 1856) have reported that penicillin treatment accelerates death from tuberculosis in the guinea-pig; and Hauduroy and Rosset ( $A n n$. 음 Inst. Pasteur, 1948, 75, 67), who have repeated these experiments, $\overline{\bar{\sigma}}$ believe such deaths to be caused simply by penicillin itself, which $\mathbb{\nabla}$ is far more toxic to guinea-pigs than to any other animal.

\section{NOTES AND COMMENTS}

Ulceration of Mucous Membranes.-Dr. James M. Canning $\vec{\omega}$ (Mitcham, Surrey) writes: With reference to the above subject, $\mathcal{O}$ on which questions and answers have previously appeared in $\frac{0}{3}$ the Journal " Any Questions?" Aug. 21, p. 408; March 15, 1947 , p. 365 ; June 15, 1946, p. 940 ; Dec. 25, 1943, p. 839), I N should like to advance a suggestion which may in some cases be of $\mathrm{cr}$ assistance to those practitioners who have had the disconcerting $\infty$ experience of endeavouring to alleviate this notorious and frequently quite intractable malady. I have observed within the past two $\overrightarrow{0}$ months a definite and very dramatic improvement in three cases of recurrent ulceration of mucous membranes from the exhibition of " benadryl." In all three cases, which were female, the ulcers appeared chiefly on the mucous membranes of the mouth, tongue, gums, and fauces, and in these sites the lesions were either identical $\rightarrow$ with or very similar to those described as recurrent aphthous or vesicular stomatitis. In each case the well-known menstrual exacerbation was noted; in two cases the lesions appeared simultaneously $\bigcirc$ on the vaginal and labial mucosa, and in one case there was a $\frac{\mathbb{D}}{3}$ co-existing idiopathic erythema multiforme affecting chiefly the $\bar{\sigma}$ anterior surfaces of both lower limbs. The patients had previously $\underline{D}$ been treated at various times with vitamins and tonics, intravenous $\vec{c}$ arsenicals, chorionic gonadotrophin, mouth washes, and local $\overrightarrow{0}$ chemical cauterization, with very disappointing results. The use of $\infty$ benadryl was instituted having in mind the recent suggestion advanced by Zondek and Bromberg (J. Obstet. Gynaec. Brit. Emp., 1947, 54, 1) that the condition may sometimes be an allergic manifestation to one or other hormone. The initial dose of the drug employed was $\frac{\bar{\partial}}{0}$ $200 \mathrm{mg}$. daily; in each case relief of local pain and tenderness was observed in 24-36 hours, and the ulcers had disappeared within 4-6 days, returning in 1-3 days after withdrawal of the drug; the $\varrho$ lesions have since been held in abeyance by an arbitrary dose of - $\overrightarrow{\vec{O}}$ at-present-50 mg. daily. In these cases familial manifestations of allergy (bronchial asthma, paroxysmal rhinorrhoea) could be traced, and it may well be that the mucosal lesions were an unusual expression of an otherwise clinically dormant allergic diathesis.

D.F.P.-With reference to our annotation (Oct. 16, p. 719) on the subject of abdominal distension and D.F.P., Messrs. Allen and Hanburys, Ltd. (Ware, Herts), inform us that they supply a sterile $0.1 \%$ solution of D.F.P. in arachis oil for intramuscular injection, 3 and 0.05 and $0.1 \%$ solutions in sterile arachis oil for ophthalmic use. The allied anticholinergic drug, tetraethylpyrophosphate (T.E.P.P.), O can also be supplied for parenteral and oral administration.

\section{Corrections}

Sir HENRY DALE writes: In my opening statement at a discussion on the physiological basis of neuromuscular disorders, at the Annual Meeting at Cambridge, a sentence on p. 890 of your issue of Nov. 20 (line 19) begins: "It is incompatible, accordingly ... etc." The $N$ passage should read: "It is compatible, accordingly

Dr. J. M. Russell, Divisional Medical Officer of Health for the $\omega$ West Riding of Yorkshire, refers to 12 cases of diphtheria notified as from Hoyland Nether Urban District ("Epidemiological Notes," Dec. 4, p. 1004). This is an error which has now been corrected by $\frac{c}{D}$ the Registrar-General. In the Weekly Returns of Infectious Diseases $\mathscr{D}$ for the week ending Nov. 13 sent to the Registrar-General the figure : 12 appeared under the heading "Diphtheria " instead of in the next column headed "Measles."

All communications with regard to editorial business should be addressed to THE $\Omega$ EDITOR, BRITISH MEDICAI JOURNAL B.M.A. HOUSE. TAVISTOCK SQUARE, EDITOR, BRITISH MEDICAL JOURNAL, B.M.A. HOUSE. TAVISTOCK SQUARE,
LONDON, W.C.1. TELEPHONE: EUSTON 2111. TELEGRAMS: Aitiology, LONDON, W.C.1. TELEPHONE: EUSTON 2111. Tondon. TELERAMS: Aitiology,
ORIGINAL ARTICLES AND LETTERS forwarded for publication are understond to be offered to the British Medical Journal alone. 8 publication are understond to be offered to the British Medical Journal alone.
Authors desiring REPRINTS should communicate with the Publishing Manager, B.M.A. House. Tavistock Square W C.1 onicate with the Publishing ADVERTISEMENTS should be addressed to the Advertisement Manager, B.M.A. House, Tavistock Square. London, W.C.1 (hours 9 a.m. to 5 p.m.). TELEPHONE: EUSTON 2111. TELEGRAMS: Britmedads, Westcent. London. MEMBERS' SUBSCRIPTIONS should be sent to the SECRETARY of the B.M.A. SCOTtish OFFICE : 7. Drumsheugh Gardens, Edinburgh. 